\title{
Utilidad de los cortes adicionales múltiples en biopsias prostáticas con atipia glandular focal
}

\author{
Arista-Nasr J*, Martínez-Mijangos O*, Martínez-Benítez B*. \\ *Departamento de Patología. Instituto Nacional de Ciencias Médica y la Nutrición, S.Z. (INCMNSZ), México DF.
}

Actas Urol Esp. 2008;32(6):594-598

\section{RESUMEN}

UTILIDAD DE LOS CORTES ADICIONALES MULTIPLES EN BIOPSIAS PROSTATICAS CON ATIPIA GLANDULAR FOCAL

Introducción: La atipia glandular focal en biopsias por punción, esta constituida por escasas glándulas que muestran criterios arquitecturales y citológicos sugestivos, pero no diagnósticos, de malignidad. Aunque éste es un problema relativamente frecuente, la ventaja de realizar niveles adicionales múltiples en estas biopsias ha sido poco estudiada. El objetivo de la presente revisión es definir cual es la utilidad de practicar cortes adicionales múltiples en biopsias por punción que muestran áreas con atipia glandular focal.

Metodos: Se seleccionaron 38 pacientes en los que se practicó biopsias por punción de la próstata que mostraron atipia glandular focal en cortes rutinarios. Todas las biopsias mostraron atipia glandular en un campo microscópico aislado. Cada caso contó originalmente con tres cortes de tres micras por laminilla. En todos los casos se practicaron tres niveles adicionales con tres cortes histológicos cada uno. Así, cada caso contó con un total de 12 cortes (3 originales y 9 adicionales).

Resultados: El promedio de edad fue de 65 años y el promedio del antígeno prostático específico de $8,4 \mathrm{ng} / \mathrm{ml}$. Los niveles adicionales permitieron establecer el diagnóstico concluyente de malignidad en nueve (22,5\%) de los 38 casos, ya que hicieron mas aparentes los criterios arquitecturales y citológicos de carcinoma prostático. En las otras 29 biopsias el área con atipia glandular se hizo menos aparente o desapareció.

Conclusiones: Los niveles adicionales son útiles para el diagnóstico de malignidad en una de cada cuatro o cinco biopsias con atipia glandular focal. Practicar cortes adicionales en estas biopsias representa un método rápido y a bajo costo que podría practicarse rutinariamente, en particular si no se cuenta con estudios inmunohistoquímicos.

Palabras clave: Carcinoma prostático. Biopsia por punción. Atipia glandular focal. Proliferación glandular atípica de acinos pequeños. Cortes adicionales.

\section{ABSTRACT \\ UTILITY OF ADDITIONAL HISTOLOGICAL SECTIONS ON PROSTATIC NEEDLE BIOPSIES WITH FOCAL GLANDULAR ATYPIA}

Introduction: Focal glandular atypia in needle prostatic biopsies consists of scarce glands with architectural and cytological features suggestive but not conclusive of malignancy. Although this is a relatively frequent finding, the advantage of carrying out additional sections has not been extensively explored. The objective of this review is to define the usefulness of performing multiple additional sections in needle biopsies showing areas with focal glandular atypia.

Methods: Thirty-eight patients in whom prostate needle biopsies were performed and showed focal glandular atypia in routine sections were selected for this study. In all biopsies there were atypia only in one isolated microscopic field. Each case originally had three cuts of three microns each per slide. In all cases three additional slides were made with three additional cuts. Thus, each case wound up with a total of 12 cuts (the original 3, and additional 9).

Results: The average age of the patients was 65 years old, and the average specific prostate antigen was $8.4 \mathrm{ng} / \mathrm{ml}$. The additional sections made it possible to establish a definitive diagnosis of malignancy in nine (22.5\%) of the 38 cases, because they made more apparent the architectural and cytological features of prostatic carcinoma. In the other 29 biopsies the area with glandular atypia was less apparent or disappeared altogether.

Conclusions: The additional sections are useful for the diagnosis of adenocarcinoma in one of every four or five needle biopsies with focal glandular atypia. Making additional cuts in these biopsies is a quick and low-cost method that could be practiced routinely, especially if immunohistochemical studies are not available.

Keywords: Prostate carcinoma. Needle biopsy. Sampling. focal glandular atypia. Atypical small glandular proliferation. Additional sections. 
$\mathrm{L}$ a atipia glandular focal (AGF), también denominada proliferación glandular atípica de acinos pequeños, ha recibido gran atención en la literatura por sus implicaciones en su pronóstico y tratamiento ${ }^{1-2}$. La falta de criterios arquitecturales, citológicos e inmunohistoquímicos suficientes en las biopsias con AGF impiden establecer un diagnóstico concluyente y con frecuencia se requiere practicar biopsias adicionales. El valor predictivo para adenocarcinoma prostático de las biopsias con atipia glandular focal varía del 40 al $50 \%$ de los casos ${ }^{1-5}$.

Aunque las ventajas de practicar niveles múltiples en biopsias prostáticas ha sido mencionada en la literatura ${ }^{6}$, solamente en un estudio se han practicado cortes adicionales en biopsias con $\mathrm{AGF}^{7}$. Nosotros hemos encontrado desde hace varios años que practicar rutinariamente cortes adicionales en biopsias con AGF puede facilitar su interpretación ya que el número de glándulas prostáticas varía notablemente de un nivel a otro ${ }^{8}$. En una revisión retrospectiva ${ }^{9}$, encontramos que en 9 de 25 biopsias (36\%), los niveles adicionales proporcionaron información útil y permitieron clasificar áreas con AGF como carcinomas focales.

El propósito de esta revisión es informar la utilidad diagnóstica de los niveles adicionales en otras 38 biopsias con el diagnóstico original de AGF.

\section{MATERIAL Y MÉTODOS}

Las biopsias de treinta y ocho pacientes con el diagnóstico de atipia glandular focal, sugestivas pero no diagnósticas de carcinoma, estudiados en el periodo 2006-2007 fueron seleccionadas. En cada caso se anoto la edad del paciente y los niveles de antígeno prostático específico.

Todos los casos contaban originalmente con una laminilla con tres cortes de 3 micras. Luego del diagnóstico de AGF, tres niveles adicionales con tres cortes adicionales por laminilla, fueron practicados. Así, cada biopsia con atipia glandular focal contó con 12 cortes (3 originales y 9 adicionales). Cada nivel adicional se denominó 1, 2 y 3 . En ningún caso se realizó estudio inmunohistoquímicos para no alterar la secuencia de los cortes.

En todas las biopsias se buscaron los siguientes cambios histológicos en los cortes adicionales: incremento o disminución del número de glándulas atípicas, aspecto infiltrativo, nucleomegalia, núcleos hipercromáticos, rigidez de luces glandulares, nuclé- olos aparentes, secreciones intraluminales basófilas, secreciones intraluminales eosinófilas, neoplasia intraepitelial prostática, mitosis, cuerpos glomeruloides, cristaloides, micronódulos colagenosos e infiltración neural. La suma de Gleason se evaluó en las áreas con mayor cantidad de glándulas observadas en los cortes adicionales. Otras áreas con carcinoma incidental no relacionadas con el área original de atipia glandular focal fueron también anotadas.

\section{RESULTADOS}

Los niveles adicionales fueron útiles en nueve de $38(22,5 \%)$ biopsias para clasificar la AGF como carcinoma. El número de glándulas en las nueve biopsias aumentó en promedio de 9 a 20 glándulas lo cual hizo más aparente el aspecto infiltrativo de la neoplasia (Figs. 1-7). En la Tabla 1 se muestra el número de glándulas encontradas en los cortes originales y el máximo de glándulas observadas en los cortes adicionales. El incremento de glándulas fue más notables en 5 de los 9 casos en el nivel 1 , en 2 de los 9 casos en el nivel 2 (Fig. 2), y 2 casos en el nivel 3 (Fig. 7). El incremento en el número de glándulas fue muy aparente en algunas biopsias y en dos casos aumentaron de 4 a 28 y de 5 a 20 glándulas neoplásicas. El incremento en el número de glándulas malignas también hizo mas aparentes otros criterios de malignidad incluyendo nucleomegalia, rigidez de luces glandulares, núcleos hipercromáticos y nucléolos aparentes. En cuatro biopsias se observaron secreciones intraluminales eosinófilas, las cuales eran escasas en los cortes originales (Figs. 4-6). El incremento de los hallazgos histológicos que facilitaron el diagnóstico de carcinoma se muestra en la Tabla 2. Aunque los criterios para el diagnóstico de carcinoma variaron de un caso a otro, la combinación de varios de ellos fue suficiente para establecer el diagnóstico de malignidad. En un caso, además del carcinoma focal se observó neoplasia intraepitelial prostática que no estaba presente en los cortes originales. En otra biopsia se encontró un cuerpo glomeruloide que tampoco era aparente en el nivele original. En ninguna de las nueve biopsias se encontraron secreciones basófilas, micronódulos colagenosos ni cristaloides. La suma de Gleason correspondió a carcinomas moderadamente diferenciados en seis casos (suma de Gleason de 6) y a carcinomas poco diferenciados en los otros tres (suma de Gleason de 7,7 y 8 ). 


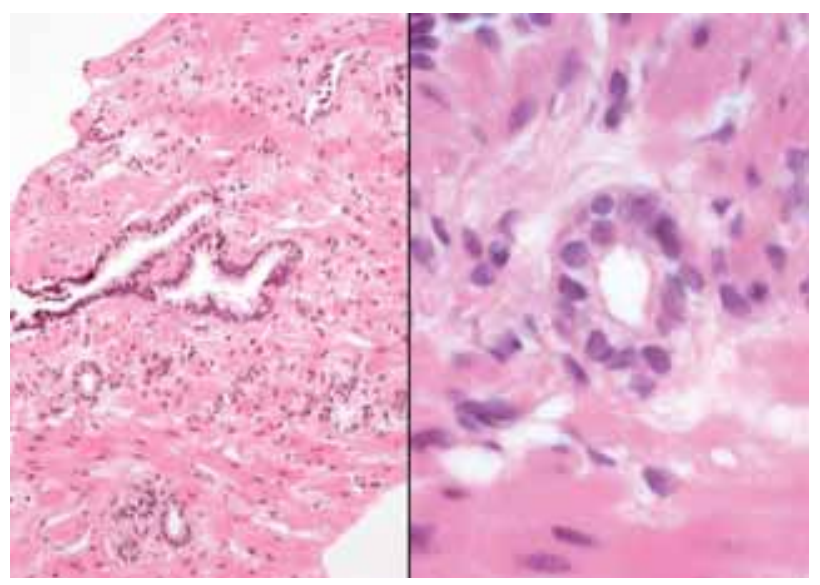

FIGURA 1. Izquierda: biopsia prostática con glándulas pequeñas aisladas en el estroma. Derecha: glándula prostática con nucleomegalia e hipercromatismo nuclear.

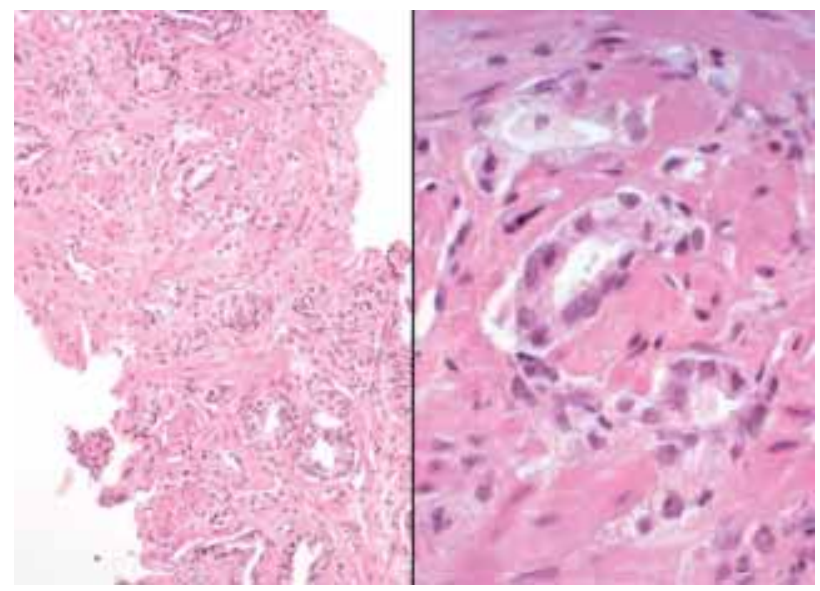

FIGURA 2. Nivel adicional 2 de la figura 1. Izquierda: hay marcado incremento de glándulas neoplásicas con aspecto infiltrativo. Derecha: glándulas malignas con infiltración estromal.

En dos biopsias, los niveles adicionales mostraron además dos microcarcinomas separados de las áreas con atipia glandular focal original. Estos carcinomas mostraron seis y ocho glándulas con aspecto infiltrante y en ambos se observaron glándulas pequeñas con nucleomegalia y/o núcleos hipercromáticos, así como nucléolos aparentes y secreción eosinófila intraluminal.

En ninguno de los 29 casos restantes se encontraron en los niveles adicionales hallazgos que apoyaran carcinoma y en la mayoría la lesión del nivel inicial se hizo menos aparente o desapareció.

\section{DISCUSIÓN}

La utilidad diagnóstica de los cortes adicionales en biopsias prostáticas con proliferaciones glandulares atípicas limitadas a campos histológicos aisla- dos ha recibido poca atención en la literatura. Es probable que un número significativo de biopsias requieran niveles adicionales si se considera que un tercio de las biopsias muestran menos de $10 \%$ de carcinoma y algunas de ellas solo habrán de mostrar escasas glándulas malignas ${ }^{10}$.

$\mathrm{Al}$ analizar la utilidad de los niveles adicionales en biopsias prostáticas benignas, Renshaw 6 encontró que $13 \%$ de aéreas con AGF y 3\% de carcinomas focales no estuvieron presentes en la primera laminilla obtenida en los cortes rutinarios. Este autor propone que al menos tres laminillas deberían ser obtenidas rutinariamente para hacer evidentes áreas con AGF microcarcinomas. En otro estudio, Reyes y Humphrey ${ }^{7}$ practicaron múltiples cortes en 100 biopsias con atipia glandular focal y neoplasia intraepitelial prostática y las compararon con 100 biopsias sin atipia. En 4 (10\%) de 40 casos con atipia glandular focal, los cambios encontrados en los

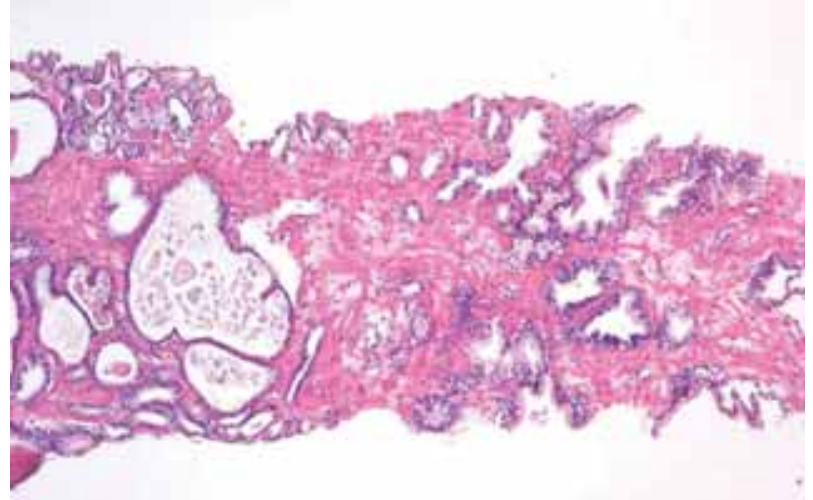

FIGURA 3. Biopsia prostática con escasas glándulas pequeñas entre glándulas benignas de mayor tamaño.

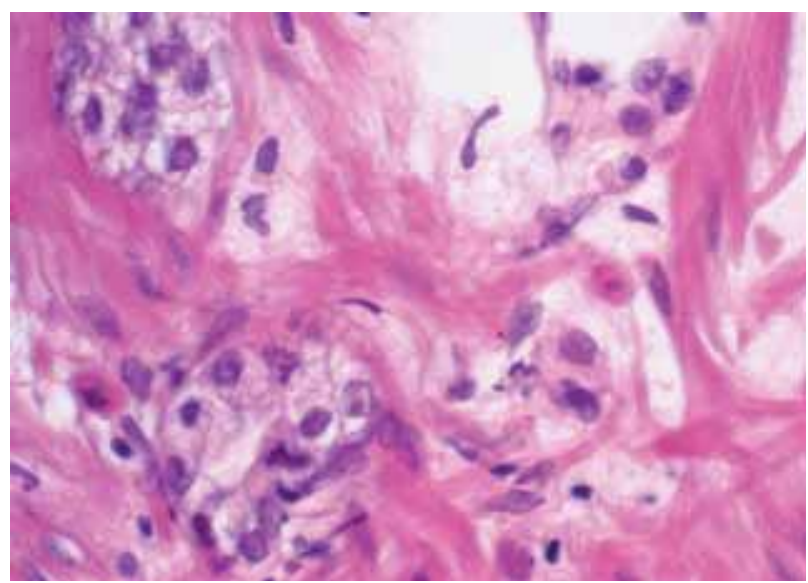

FIGURA 4. Detalle de glándulas pequeñas. A pesar de los cambios por artificio se observa nucleomegalia y nucléolos aparentes. 


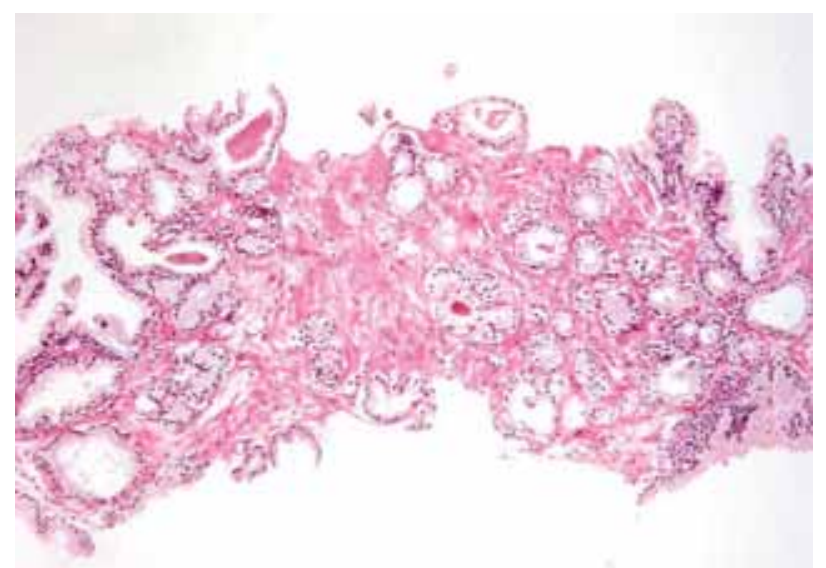

FIGURA 5. Nivel adicional 2 en biopsia de figura 3 y 4. Se observan glándulas de mediano y pequeño tamaño con aspecto infiltrante. Algunas muestran secreción intraluminal.

niveles adicionales fueron suficientes para diagnosticar carcinoma. Estos autores recomiendan obtener al menos una laminilla extra con múltiples cortes adicionales de tres micras luego de que se ha detectado un área con atipia glandular focal. En las biopsias con neoplasia intraepitelial prostática los niveles adicionales no modificaron el diagnóstico.

En una revisión que llevamos a cabo en $1998^{9}$, encontramos que practicar tres niveles adicionales fue útil para el diagnóstico de carcinoma en 25 (36\%) de nueve casos por diversas razones que incluyeron: un incremento en el número de glándulas que mostraban criterios citológicos y arquitecturales convincentes de malignidad, presencia de otras áreas con carcinoma que no estaban presentes en los cortes originales, y un caso que mostró invasión perineural que no era evidente en el corte original.

En el presente estudio los resultados fueron semejantes y encontramos que los niveles adicionales fueron útiles para diagnosticar biopsias con atipia glandular focal como carcinomas en las 9 de $38(22,5 \%)$ biopsias. El incremento de glándulas atípicas hizo mas aparente el aspecto infiltrativo de la neoplasia y también se hicieron mas aparentes varios criterios citológicos de carcinoma prostático.

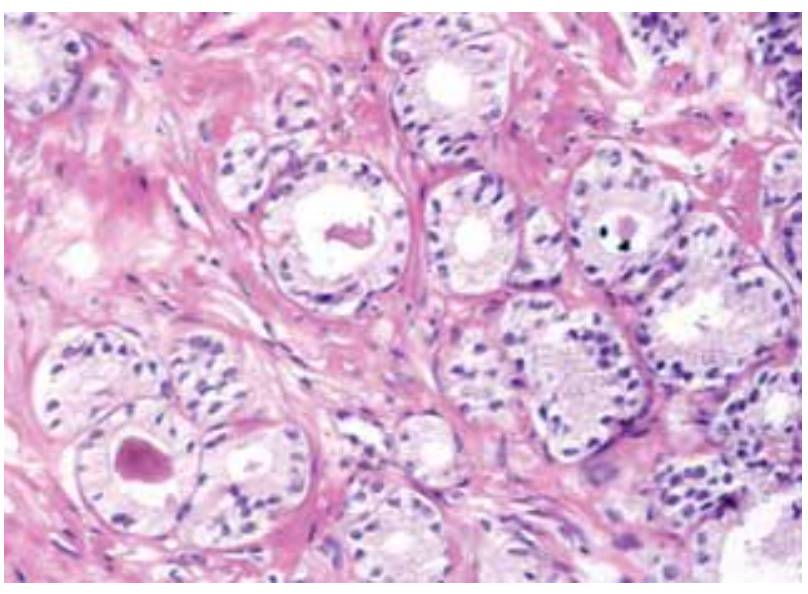

FIGURA 6. Detalle de la figura 5. Glándulas con luces rígidas, secreción intraluminal y nucléolos.

Si se consideran las 25 biopsias con AGF de la revisión previa ${ }^{9}$ y las 38 biopsias con AGF del presente estudio, el porcentaje de utilidad diagnóstica de los niveles adicionales en nuestra experiencia es aproximadamente del 30\%. Debe enfatizarse que es imposible saber cuales son las biopsias con AGF que habrán de mostrar un mayor número de glándulas en los niveles adicionales. Por esta razón es necesario realizar cortes adicionales en todas las biopsias con atipia glandular focal.

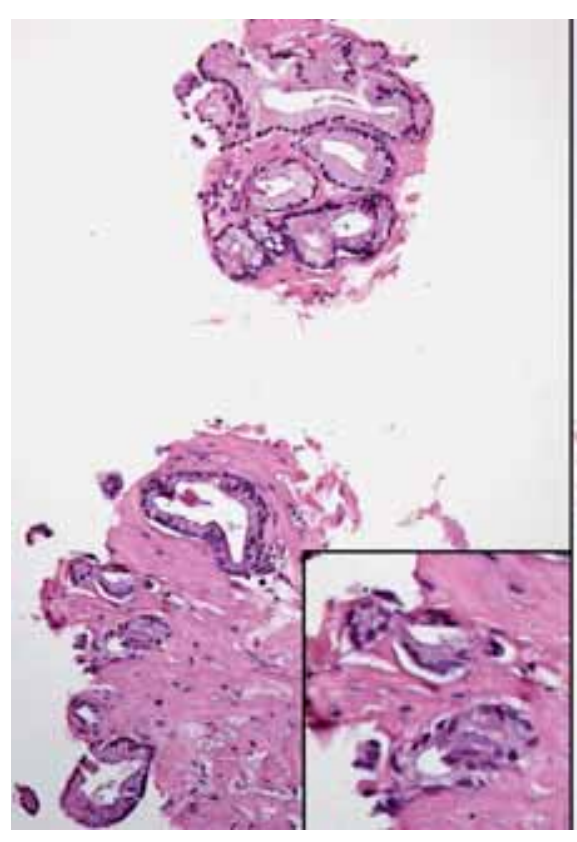

FIGURA 7. Izquierda: nido de glándulas atípicas dispuestas estrechamente entre sí. Derecha: Nivel adicional 3. Glándulas neoplásicas infiltrando el estroma. Algunas muestran nucléolos y nucleomegalia.

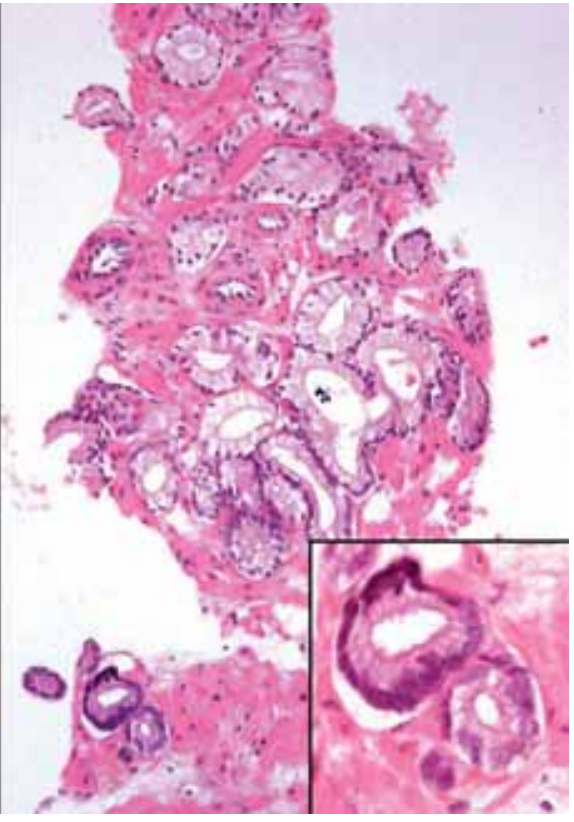
(n) 
Tabla 1. Número de glándulas en cortes originales y en adicionales en nueve casos con atipia glandular focal

\begin{tabular}{lccc}
\hline & $\begin{array}{c}\text { No. de glándulas } \\
\text { en corte } \\
\text { original }\end{array}$ & $\begin{array}{c}\text { No. de glándulas } \\
\text { en cortes } \\
\text { adicionales* }\end{array}$ & $\begin{array}{c}\text { Suma de } \\
\text { Gleason** }\end{array}$ \\
\hline $1-$ & 10 & 12 & 7 \\
$2-$ & 5 & 20 & 6 \\
$3-$ & 4 & 28 & 6 \\
$4-$ & 8 & 26 & 7 \\
$5-$ & 6 & 10 & 6 \\
$6-$ & 5 & 10 & 6 \\
$7-$ & 5 & 20 & 6 \\
$8-$ & 25 & 30 & 6 \\
$9-$ & 14 & 25 & 8 \\
Promedio: & 9 & 20 & \\
\hline
\end{tabular}

* Se anotan el número máximo de glándulas encontradas en cualquiera de los cortes adicionales.

**La suma de Gleason se evaluó en el corte con mayor cantidad de glándulas.

Tabla 2. Niveles adicionales en nueve biopsias con atipia glandular focal. Incremento de hallazgos histologicos*

Patrón infiltrativo

Rigidez de luces glandulares

Nucleomegalia

Núcleos hipercromáticos

Nucléolos aparentes

Secreciones intraluminales eosinófilas

Cuerpos glomeruloides

Neoplasia intraepitelial prostática

*El incremento de estos criterios varió de un caso a otro.

Aunque en este estudio no se practicaron estudios inmunohistoquímicos para no alterar la secuencia de los cortes adicionales, la utilidad de las citoqueratinas de alto peso molecular y la coenzima A racemasa ha sido ampliamente reconocida en la literatura. Algunos autores han propuesto realizar los estudios inmunohistoquímicos en niveles adicionales alternando con cortes convencionales teñidos con hematoxilina y eosina para analizar una mayor cantidad de glándulas atípicas ${ }^{11}$. Debe considerarse sin embargo, que muchos laboratorios no cuentan con técnicas inmunohistoquímicas. En tales circunstancias, los niveles adicionales podrían representar una alternativa simple y a bajo costo que contribuya a facilitar la interpretación de las biopsias prostáticas con atipias glandulares focales.

\section{REFERENCIAS}

1. Cheville JC, Reznicek MJ, Bostwick DG. The focus of "atypical glands, suspicious for malignancy" in prostatic needle biopsy specimens: incidence, histologic features, and clinical follow-up of cases diagnosed in a community practice. Am J Clin Pathol. 1997;108(6):633-640.

2. Iczkowski KA, Mc Lennan GT, Bostwick D. Atypical small acinar proliferation suspicious for malignancy in prostate needle biopsies: clinical significance in 33 cases. Am J Surg Pathol. 1997;21(12): 1489-1495.

3. Girasole CR, Cookson MS, Putzi MJ, Chang SS, Smith JA Jr, Wells N, et al. Significance of atypical and suspicious small acinar proliferations, and high grade prostatic intraepithelial neoplasia on prostate biopsy: implications for cancer detection and biopsy strategy. J Urol. 2006;175(3 Pt1):929-933.

4. Chan TY. Epstein JI. Follow-up of atypical prostate needle biopsies suspicious for cancer. Urology. 1999;53(2):351-355.

5. Iczkowski KA, Bostwick DG. Criteria for biopsy diagnosis of minimal volume prostatic adenocarcinoma: analytic comparison with nondiagnostic but suspicious atypical small acinar proliferation. Arch Pathol Lab Med. 2000;124(1): 98-107.

6. Renshaw AA. Adequate tissue sampling of prostate core needle biopsies. Am J Clin Pathol. 1997;107(1):26-29.

7. Reyes AO, Humphrey PA. Diagnostic effect of complete histologic sampling of prostate needle biopsy specimens. Am J Clin Pathol. 1998;109(4):416-422.

8. Bonney WW, Robinson RA, Lachenbruch PA, Culp DA. Yield of cancer tissue from prostatic needle biopsy. Urology. 1987;29(2):153-156.

9. Arista-Nasr J, Keirns C. The focus of "atypical glands, suspicious for malignancy" in prostatic needle biopsy specimens. Am J Clin Pathol. 1998; 110(3):409.

10. Bostwick DG. Gleason grading of prostatic needle biopsies. Correlation with grade in 316 matched prostatectomies. Am J Surg Pathol. 1994;18(8):796-803.

11. Humphrey PA. Prostate Pathology, 2003: pp223. ASCP Press, Hong Kong,

Correspondencia autor: Dr. J. Arista-Nasr

Departamento de Patología. Instituto Nacional de Ciencias

Médicas y de la Nutrición S.Z. Vasco de Quiroga, 15, Tlalpan,

1400 Mexico

Tel.: (+52-5) 5487-0900, exts. 2186 y 2184

E-mail autor: brauliomb77@yahoo.com.mx / pipa5@hotmail.com

Información artículo: Original - Cáncer de próstata

Trabajo recibido: noviembre 2007

Trabajo aceptado: enero 2008 